\title{
Atypical presentation of sporotrichosis: report of three cases
}

\author{
Melissa Orzechowski Xavier ${ }^{[1],[2], ~ L a u r a ~ R i f f e l ~ B i t t e n c o u r t ~}{ }^{[2],[3],}$ \\ Cláudio Moss da Silva ${ }^{[1],[4]}$, Roseli Stone Vieira ${ }^{[4]}$ and Hugo Cataud Pacheco Pereira ${ }^{[1]}$
}

[1]. Área Interdisciplinar de Ciências Biomédicas, Área de Clínica Médica, Faculdade de Medicina, Universidade Federal do Rio Grande, Rio Grande, RS. [2]. Laboratório de Micologia, Faculdade de Medicina, Universidade Federal do Rio Grande, Rio Grande, RS. [3]. Curso de Medicina, Universidade Federal do Rio Grande, Rio Grande, RS. [4]. Serviço de Infectologia, Hospital Universitário Dr. Miguel Riet Corrêa Jr, Universidade Federal do Rio Grande, Rio Grande, RS.

\begin{abstract}
Sporotrichosis occurs after fungal implantation of Sporothrix spp. in the skin, and is the main subcutaneous mycosis in Latin America. Here we describe three atypical cases of the disease. The first case report an extra-cutaneous occurrence of the disease with joint infection; the second one describes a patient with bilateral lymphocutaneous form of sporotrichosis; and the third shows a zoonotic cutaneous case with the development of an erythema nodosum as a hypersensitivity reaction. These cases show the disease importance on the region and the necessity of fungal culture to the diagnosis confirmation.
\end{abstract}

Keywords: Sporothrix sp. Septic arthritis. Erythema nodosum. Itraconazole.

\section{INTRODUCTION}

Sporotrichosis, acquired by inoculation of the dimorphic fungus Sporothrix spp., is the main subcutaneous mycosis in Brazil. Clinically the typical presentation of sporotrichosis is a reddish lesion that appear near to the inoculation site and which tends to progress to erithematous nodules and ulcers with new similar lesions ascending in lymphatic vessels ${ }^{1,2}$.

Although ascending nodular lymphangitis is the commonest clinical presentation, the disease can manifest with distinct characteristics, which turns the diagnosis more difficult. Here we describe three atypical cases of sporotrichosis diagnosed in 2010 and 2011 at the University Hospital of Universidade Federal do Rio Grande (HU-FURG), Rio Grande, State of Rio Grande do Sul, Brazil. This study was approved by the Ethic Committee of the Institution (CEPAS-FURG 175/2011).

\section{CASE REPORT}

A male patient, 51-years-old, fisherman, was admitted with a complaint of pain and swelling on his right knee that had started one year before, since the occurrence of a local bruising trauma in a wood boat, but without skin penetration. Serology for human immunodeficiency virus (HIV) and hepatitis $\mathrm{C}$ virus (HCV) were negative, but the patient suffered of diabetes, and was drinker and smoker in the past (two years and seven years before, respectively). Previous ultrasonography suggested septic arthritis, and patient was treated with surgical procedure and cefalotin without clinical resolution. In that occasion, laboratorial

Address to: Dra. Melissa Orzechowski Xavier. Lab. Micologia/FM/FURG. Campus Saúde, Visconde de Paranaguá 102, 96201-900 Rio Grande, RS, Brasil. Phone: 5553 3233-8871

e-mail: melissaxavier@furg.br

Received in 08/02/2012

Accepted in 21/05/2012 exams of the sinovial fluid showed no microorganism growth in bacterial and fungal culture. Clinical examination revealed pain with high intensity, difficulty walking, and flogistic signs on the affected joint (Figure 1). Ultrasound imaging showed an extensive suffusion with a viscous fluid and suprapatellar bursa distension. Suspecting of a septic arthritis reactivation an arthrocentesis was done. Cytology of synovial fluid revealed a count of 800 erythrocytes $/ \mathrm{mm}^{3}$ and of 3,200 leukocytes $/ \mathrm{mm}^{3}$ with $53 \%$ of lymphocytes and $47 \%$ of segmented neutrophil. Biochemical examination was not done due to the synovial

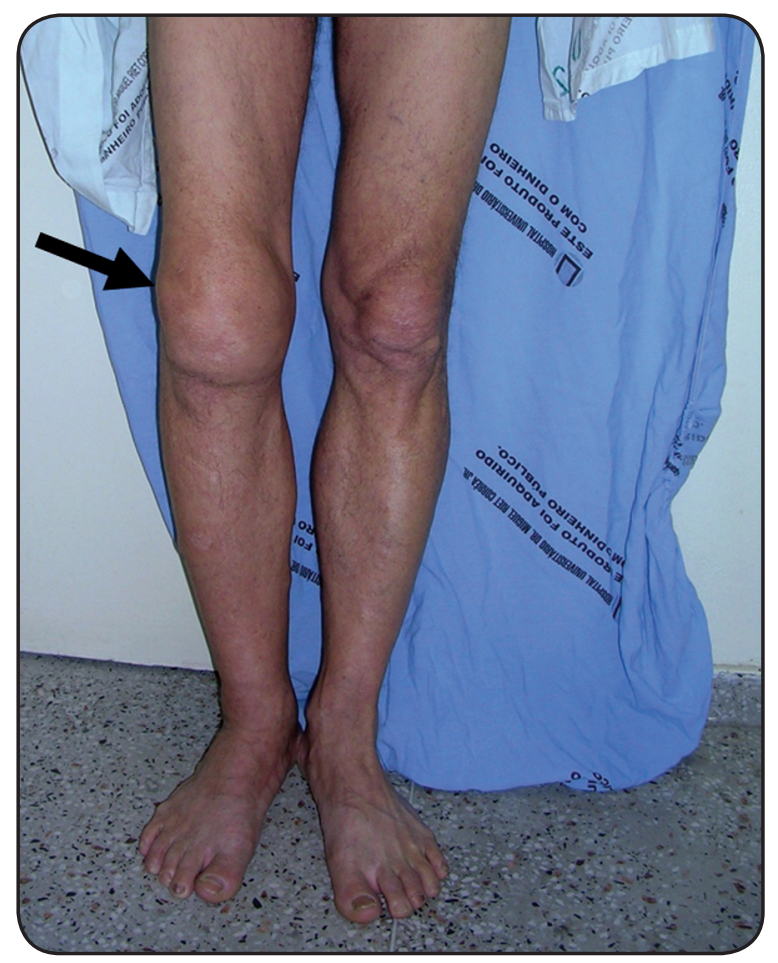

FIGURE 1 - Black row showing swelling in the right knee of the patient with monoarthritis by Sporothrix sp. (Case 1). 
thickening. At the microbiology lab, none bacterial growth was detected, but a great number of fungal colonies grew in Sabouraud agar. The characteristics of the colonies in Sabouraud agar at $25^{\circ} \mathrm{C}$ and in Brain-Heart Infusion (BHI) agar at $37^{\circ} \mathrm{C}$ associated with its micromorphology allowed us to confirm the diagnosis of a Sporotrhix sp. articular infection. The patient was scheduled to treat his arthritis with itraconazole $(200 \mathrm{mg}$ twice a day - BID) for one year. After six months of treatment, it was observed a significant improvement of his articular symptoms.

\section{Case 2}

Male patient, 43 years-old, soldier, was admitted in the HU-FURG ambulatory showing ulcerated, papular and nodular lesions, with violaceous borders in both arms and distributed by the lymphatic pathway. The patient reported the practice of exercises crawling and rolling on the ground with vegetation. This professional practice occurred one week before the appearance of the first lesion on his left arm, which progressed following the lymphatic vessels and also appeared in the right arm. About ten lesions $(1-3 \mathrm{~cm})$ in each arm were observed in the clinical examination, some with intact skin and others ulcerated with exudates (Figure 2). Regional lymphadenomegaly was also detected. According to the patient, the lesions had an evolution about three or four months without clinical resolution with previous antibacterial treatment. Clinical suspicion of bilateral lymphocutaneous sporotrichosis was done and patient was submitted to a skin biopsy. Histopathological evaluation showed mixed multifocal inflammatory process without any identifiable microorganism or malignity evidence. At mycology lab a great number of fungal colonies grew in Agar Sabouraud and were phenotipically identified as Sporothrix sp., confirming the initial clinical suspicion. Itraconazole was prescribed at $200 \mathrm{mg} /$ day for three months. Patient did not return to the evaluation of his clinical evolution.

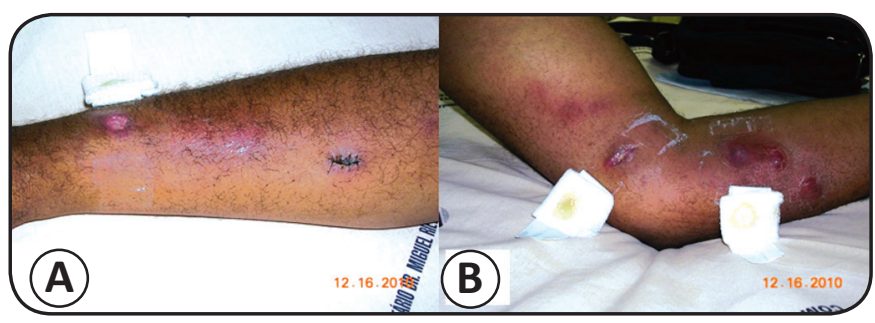

FIGURE 2 - Ulcerated, erythematous nodular lesions in right (A) and left (B) arm of the patient with bilateral lymphocutaneous sporotrichosis (Case 2).

\section{Case 3}

Female patient, 28 years-old, student, referred the appearance of a skin lesion about 15 days after a scratch of a cat with sporotrichosis. Physical examination revealed a $3 \mathrm{~cm}$ pustular lesion with violaceous borders in the trauma region in her forearm, associated with a more recent $2 \mathrm{~cm}$ lesion, nodular, erythematous and firm, following the lymphatic vessel. After eight days of the initial skin lesions at forearm, multiple painfull reddish nodules appeared in her both legs (Figure 3), featuring a typical case

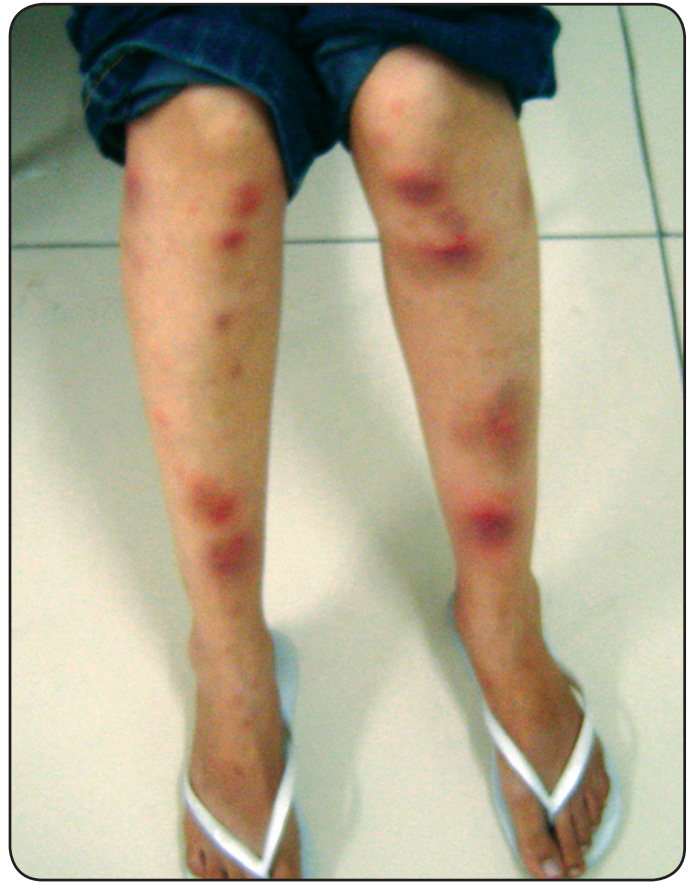

FIGURE 3 - Skin rash due to a hypersensitivity reaction in a zoonotic case of sporotrichosis (Case 3).

of erythema nodosum. Histopathology of the biopsy of the ulcerated lesion in forearm showed an inflammatory process with great number of multinucleated foreign body giant cells, and it cultive resulted in Sporothrix sp. growth, confirming the diagnosis of sporotrichosis. The immunological condition of the patient was investigated due to the probability of a fungal dissemination, but immunossupression was ruled out. Nonsteroidal antiinflammatory drugs resolved the eryhtema nodosum in a few weeks, and treatment with itraconazole $200 \mathrm{mg} /$ day for six months resulted in a clinical cure.

\section{DISCUSSION}

The clinical presentation of sporotrichosis described here, as septic arthritis, bilateral lymphatic lesions and hypersensitivity reaction to Sporothrix sp. infection, are rare with scarce reports in the literature.

Cases of osteoarticular sporotrichosis are frequently characterized as chronic monoarthritis, mainly affecting the $\mathrm{knee}^{3-5}$, as described in our patient 1 . Although less common, the involvement of elbows, ankles and wrists can also occur ${ }^{5}$. The two hypotheses about the source of infection in our case 1 are: fungal inoculation by the initial traumatic lesion occurred in a wooden boat as reported by the patient; or articular contamination during the surgical procedures performed to diagnostic evidences ${ }^{6}$. In fact, a case of sporotrichosis after the onset of infusion drug for the treatment of arthritis has been described in the literature as a result of non-sterile handling of the lesion ${ }^{6}$. However, the non-regression of the lesion in our patient, even with the use of antibiotics, anti-inflammatory and surgical procedures, suggests the fungal involvement since the beginning, probably acquired by the trauma reported. Similar 
monoarthritis case as the only evidence of sporotrichosis without the presence of a skin lesion has been previously reported in the literature ${ }^{4}$, and authors also did not found a specific source of infection.

Arthritis due to Sporothrix sp. is often associated with immunosuppressed patients, especially those with acquired immunodeficiency syndrome (AIDS). In our case 1, patient despite being negative for HIV, had a history of chronic alcoholism and diabetes, factors also cited as a predisposition to the onset of extra-cutaneous sporotrichosis ${ }^{1,5}$. Differential diagnosis of monoarthritis include other infectious causes, and noninfectious, as hypothyroidism, amyloidosis, multiple myeloma, paraneoplastic syndrome, among others ${ }^{4}$.

According to the majority of reported cases of bilateral sporotrichosis, this presentation may occur by self-inoculation, multiple inoculations or re-infection ${ }^{7}$. The crawling exercise in soil with decaying vegetation, performed by our patient 2 prior to the onset of the bilateral lymphocutaneous lesions, suggests that there has been simultaneous multiple inoculations of the fungus into the dermis through microtrauma by plant material, since this is the natural habitat of Sporothrix sp., similar to that reported by other authors ${ }^{7,8}$. The bilateral presentation of sporotrichosis is described as atypical and rare, although it have already been reported in children by zoonotic transmission ${ }^{9}$ or by trauma with plants in adults ${ }^{7,8}$. Differential diagnosis of this clinical presentation of sporotrichosis include cutaneous tuberculosis, atypical mycobacterial infection, prurigo, nocardiosis, and cutaneous leishmaniasis in its sporothrichosis-like clinical form ${ }^{1,7}$.

Hypersensitivity reactions to infection by Sporothrix sp. as occurred in our case 3 may present themselves as different clinical manifestations. Case of a reactional episode of polyarthritis was described in an immunocompetent patient with lymphocutaneous sporotrichosis that showed the involvement of ankles and elbows ${ }^{10}$. Three cases of erythema multiforme (EM) associated with Sporothrix sp. infection had been described in adult patients that developed a skin rash after 7-30 of the primary infectious cutaneous ${ }^{11}$. And, similar to our case 3, hypersensitivity fungal triggering erythema nodosum (EN) associated with sporotrichosis was first described in 2002, in three patients in Rio de Janeiro ${ }^{12}$. According to our case reported, the lesions of EN described in the literature may arise after 7 to 40 days of the initial injury ${ }^{12}$.

Although few cases of hypersensitivity to Sporothrix sp. infection are reported in the literature, as in our case 3, all of them ${ }^{10-12}$ occurred through zoonotic transmission from sick cats. A hypothesis that could explain this association is the constant and intense stimulation of the immune system of patients when they are in touch with cats with sporotrichosis ${ }^{11,12}$. This could occur due to the fact that this species tends to have a great amount of fungal propagules in their lesions, triggering an intense exposure of humans to fungus antigens and even culminating with subclinical infection and reinfection, causing hypersensitivity ${ }^{2,11,12}$.

We must remark that the gold standard for diagnosis of sporotrichosis in any clinical presentation is mycological culture for fungi isolation and identification ${ }^{1}$, as occurred in the three cases reported here. The fungal culture is required because the sporotrichosis lesions in humans tend to have few yeast cells which provide a difficulty in observe them either in cytological or histopathological examination ${ }^{1,4}$.

However, the difficulty of diagnosis of atypical sporotrichosis described in this study are also frequently reported in the literature, either in bilateral case of sporotrichosis ${ }^{79}$ or in the osteoarticular involvement ${ }^{3,4}$, especially given the numerous other causes more commonly associated with, which are raised as the main diagnostic hypotheses ${ }^{2,4}$. In this scenario, it is common the occurrence of a late diagnosis, and, especially in relation to osteoarticular involvement, the delay in identifying the etiology of the injury can lead to unfavorable prognosis and may result in loss of function of the affected area ${ }^{4,5}$, similar to that observed in our patient that showed signs of muscle atrophy by disuse as a consequence of chronic difficulty in walking.

In conclusion, the three reported cases of atypical sporotrichosis described here emphasize the disease importance in the region, and highlight the need to include it as differential diagnosis even in cases of non-classic lesions of the disease. Thus, fungal culture will be conducted leading in the diagnosis confirmation and favoring the prognosis through an early and correct treatment.

\section{REFERENCES}

1. Kauffman CA, Bustamante B, Chapman SW, Pappas PG. Clinical Practice Guidelines for the Management of Sporotrichosis: 2007 Update by the Infectious Diseases Society of America. Clin Infect Dis 2007; 45:1255-1265.

2. Lopes-Bezerra LM, Schubach A, Costa RO. Sporothrix schenckii and Sporotrichosis. Anais Acad Bras Ciências 2006; 78:293-308.

3. Appenzeller S, Amaral TN, Amstalden EMI, Bertolo MB, Marques Neto JF, Samara AM, et al. Sporothrix schenckii infection presented as monoarthritis: report of two cases and review of the literature. Clin Rheumatol 2006; 25:926-928.

4. Costa RO, Mesquita KC, Damasco PS, Bernardes-Engemann AR, Dias CMP, Silva IC, et al. Infectious arthritis as the single manifestation of sporotrichosis: Serology from serum and synovial fluid samples as an aid to diagnosis. Rev Iberoam Micol 2008; 25:54-56.

5. Howell SJ, Toohey JS. Sporotrichal arthritis in south central Kansas. Clin Orthop Relat Res 1998; 346:207-214.

6. Gamo R, Aguilar A, Cuétara M, Gonzalez-Valle O, Houmani M, Martín L, et al. Sporotrichosis Following Mesotherapy for Arthrosis. Acta Derm Venereol 2007; 87:430-431.

7. Haruna K, Shiraki Y, Hiruma M, Ikeda S, Kawasaki M. A case of lymphangitic sporotrichosis occurring on both forearms with a published work review of cases of bilateral sporotrichosis in Japan. J Dermatol 2006; 33:364-367.

8. Alves SH, Aurélio PL, Tecchio MZ, Zuchetto A, Schirmer R, Santurio JM. Subcutaneous bilateral sporotrichosis: A rare presentation. Mycopathologia 2004; 158:285-287.

9. Borrego JAB, Mayorga J, Tarango-Martínez VM. Esporotricosis linfangítica bilateral y simultánea. Rev Iberoam Micol 2009; 26:247-249.

10. Orofino-Costa R, Bóia MN, Magalhães GAP, Damasco PS, Bernardes-Engemann AR, Benvenuto F, et al. Arthritis as a hypersensitivity reaction in a case of sporotrichosis transmitted by a sick cat: clinical and serological follow up of 13 months. Mycoses 2009; 53:81-83.

11. Galhardo MCG, Barros MBL, Schubach AO, Cuzzi T, Schubach TMP, Lazéra MS, et al. Erythema multiforme associated with sporotrichosis. Eur Acad Dermatol Venereol JEADV 2005; 19:507-509.

12. Galhardo MCG, Schubach AO, Barros MBL, Blanco TCM, Cuzzi-Maya T, Schubach TMP, et al. Erythema nodosum associated with sporotrichosis. Int $\mathrm{J}$ Dermatol 2002; 41:114-116. 